\title{
Orientación y diversidad: Por una educación valiosa para todos y todas
}

\section{Counseling and Diversity: Valuable Education for All}

\author{
Cindy Artavia Aguilar ${ }^{1}$ \\ División de Educación para el Trabajo, Centro de Investigación y Docencia en Educación \\ Universidad Nacional \\ Heredia, Costa Rica \\ cartaviaa@gmail.com \\ Manuel Arturo Fallas Vargas ${ }^{2}$ \\ División de Educación para el Trabajo, Centro de Investigación y Docencia en Educación \\ Universidad Nacional \\ Heredia, Costa Rica \\ gutfallas@hotmail.com
}

Recibido 15 de marzo de 2012 . Corregido 10 de noviembre de 2012 • Aceptado 07 de noviembre de 2012

Resumen. En una sociedad altamente cambiante y compleja, llena de desafíos en todos los ámbitos, es fundamental plantearnos nuevos paradigmas educativos que respondan a las necesidades de las diversas poblaciones. En este escrito presentamos una reflexión sobre la disciplina de la orientación y el propósito de asumir este reto con responsabilidad, con ética y con un posicionamiento crítico de las realidades sociales. Se reflexiona sobre la capacidad de crear espacios inclusivos para todos los seres humanos; donde se retome la educación inclusiva como un paradigma emergente dentro de la cultura de la diversidad y se refleje su aplicación, de forma directa, en la cotidianidad de esta labor.

Palabras claves. Diversidad, Orientación, educación, cotidianidad y discurso.

Abstract. In a rapidly changing and complex society full of challenges in all areas, it is vital to introduce new educational paradigms to meet the needs of diverse populations. This paper analyzes the necessity to face this challenge with responsibility, on an ethical framework and through critical thinking on the social realities, bringing attention to Counseling, as a discipline. It discusses the capacity to create inclusive opportunities for all, where inclusive education is seen as an emerging paradigm within a culture of diversity that can applied in our everyday activities.

Keywords. Diversity, counseling, education, everyday activities, discourse.

Bachiller y licenciada en Orientación, Universidad Nacional. Máster en Pedagogía con énfasis en la Diversidad de los Procesos Educativos, Universidad Nacional. Académica, investigadora y extensionista de la Universidad Nacional.

2 Bachiller y licenciado en Orientación, Universidad Nacional. Magíster en Psicopedagogía, Universidad De La Salle. Doctor en Sexualidad y Relaciones Interpersonales, Universidad de Salamanca. Académico, investigador y extensionista de la Universidad Nacional. 
Conceptualizar el término diversidad no es tarea fácil, especialmente cuando implica tanto dinamismo, compromiso y coherencia. El mundo, los seres humanos y todo lo que ello contemple en sí mismo es diverso, es una diversidad innegable y que configura toda la realidad de nuestras acciones en la sociedad.

El término diversidad no es sinónimo de diferencias, por el contrario, se enriquece con estas. Se entiende como un proceso dinámico que responde a diferentes ámbitos del ser humano; a grupos culturales o étnicos; a género, clase social, estilos y formas particulares de aprender; a personas con discapacidad o sobre capacitadas, entre otras características personales y sociales. Es "(...) una característica propia de la humanidad consistente en 'desarrollar infinitas respuestas ontogenéticas en el contexto unitario de la filigénesis' (Essomba, 2003) (...) esto significa que, en principio, todos los seres humanos compartimos un mismo bagaje (...)" (Essomba, 2006, p. 54).

La diversidad implica el reconocimiento del ser humano, por tanto, no puede ser naturalizada ni negada. Este reconocimiento es visible en la cotidianidad, la cual es el reflejo directo de ideologías y culturas. Las manifestaciones de inclusión, igualdad y respeto a la diversidad se descubren en el lenguaje, creencias, afectos, etc. de cada manifestación humana (cultura). Sin embargo, el término está en constante riesgo por la razón de que "(...) vivimos en un escenario social complejo. El imperante modelo de globalización borra cada vez más las diferencias e instala cierta uniformidad y homogeneidad en los códigos de comunicación (...)" (Devalle y Vega, 1999, p. 23). Por el contrario, desde el enfoque de las diferencias, además de ser una realidad, debería ser considerada, en la práctica, como un elemento de enriquecimiento.

Como podemos ver, el concepto de diversidad es amplio y complejo, y nos involucra a "todos" en un "todo" de convivencia humana.

El término diversidad debe ser acuñado de forma crítica en el quehacer educativo, porque educar en la diversidad y para espacios diversos no es un añadido o complemento de la educación, sino que está en las mismas entrañas; en la diversidad, el discurso y la práctica se deben fusionar, de manera que no resulte una imposición sin sentido al sistema educativo nacional.

Esta visión abre el espacio para reflexionar y actuar sobre una construcción diferente de pedagogía en la diversidad, la que, por ende, nos plantea paradigmas distintos, modelos o posiciones críticas, de comprensión, significativos, cooperativos, revolucionarios, de amor, entre otros; donde el fin primordial es el bienestar integral del ser humano, y una educación acorde con los contextos sociales que demanda respuestas claras y concretas de la misma educación social.

La educación debe ser sustentada y validada desde la diversidad, por muchas razones, dentro de las que cabe destacar que hemos caído en enfoques erróneos e injustos, bastante habituales como la homogenización y uniformidad, donde se ofrece lo mismo a todos y todas; o aquellos orientados a las dificultades vistas como resultado de insuficiencias 0 limitaciones individuales. Ha generado, como resultado, la segregación estudiantil, la cual se opone al desarrollo personal y social, por tanto, empobrece el aprendizaje. También implica 
la adaptación del estudiante a modelos determinados correspondientes de una visión única y aislada de la realidad; potenciar unos patrones de conducta y unos aprendizajes frente a otros $y$, por ende, favorecer determinados sectores de población, en detrimento de otros. En síntesis, una visión que impide la búsqueda de alternativas de transformación basadas en la interacción, en el conocimiento compartido del mundo y en la negación.

Los orientadores y orientadoras, como agentes de cambio social y personal, debemos tener en nuestra labor un posicionamiento claro y consciente, que no es, ni puede ser, aséptico. No ser conscientes de ello significa, lejos de ser neutrales, asumir acríticamente la cultura social dominante.

Aun siendo una tarea difícil encontrar opciones de diversidad que partan del mismo escenario pedagógico, existen en nuestro sistema educativo formal experiencias y apoyos a la educación que permiten variar las respuestas e interpretaciones desde lo más cotidiano y cercano al estudiante, para darle soluciones al mismo ser humano. Estas nacen del seno de la misma interpretación de la realidad, por un lado, como posibles acciones y propuestas transformadoras de la visión de diversidad y, por otro, la opción de convertir la diversidad en un reto para algunos grupos sociales se considera como un problema. Este reto implica una nueva visión y revisión de los contextos educativos en los que nos movilizamos cotidianamente, como los espacios de aula, centro, entorno, recursos, personal y relaciones interpersonales, entre otros espacios de interacción pedagógica que transforman y dan sentido a las acciones educativas y cotidianas de nuestra vida, las cuales, en muchos momentos son rutinarias y carecen de sentido para el estudiantado, docentes y gestores de la educación.

Ante este escenario, es fundamental que todas las personas involucradas en estos procesos de enseñanza-aprendizaje tengamos clara la finalidad de la educación en nuestro país y la intencionalidad del sistema educativo en cada etapa, nivel o ciclo en particular. Cada una de estas etapas o ciclos deben tener sentido entre sí mismos, y manifestar una relación lógica y coherente, tanto horizontal como verticalmente dentro del currículo nacional y con pertinencia internacional; esta relación tiene sentido no solo desde la alternativa académica, sino desde lo social y cultural, lo que también implica que tendrían que clarificarse y definirse en función de las capacidades y habilidades personales, cognitivas y académicas, con el objetivo primordial de brindarle al colectivo estudiantil estrategias, recursos y herramientas para enfrentarse o posicionarse ante la sociedad de forma integral, crítica y con sentido de acción y justicia social.

Esto supone replantearnos, por ejemplo, la significatividad de la secuencia de contenidos ofrecidos con base en interrogantes como: ¿Qué o quién marca la estructuración del contenido dentro del currículo?, o bien, ¿quiénes quedan por fuera del sistema educativo, porque su cultura no está dentro del contenido nacional o se desvaloriza frente a lo imponente? Desde este planteamiento reflexivo se intenta establecer vínculos significativos entre la realidad del estudiante y la escuela, de manera tal que el ser humano forme parte activa de la construcción del saber: del contenido escolar, la didáctica y toda estrategia pedagógica; como bien lo menciona Freire (1973), la relación entre el estudiante, la naturaleza y la cultura "no puede ser reducida a un estar frente, o a un estar sobre, o a un estar para (...) puesto que debe ser un estar con ellos, como sujetos de cambio" (p. 63). 
Como mencionan Savater, citado por Devalle y Vega (1999), muchas veces resulta comprensible el "(...) temor ante una enseñanza cargada de contenidos ideológicos, ante una escuela más ocupada en suscitar fervores y adhesiones inquebrantables que en favorecer el pensamiento crítico autónomo" (p. 25). Pero no se puede minimizar la posibilidad de crear oportunidades y espacios educativos que consideren la diversidad en cualquier inicio de la educación, para garantizar la homogeneidad en los resultados obtenidos, respetando la individualidad del ser humano y su construcción personalsocial-cultural desde sus propios constructos pedagógicos.

El reto de una educación inclusiva como un paradigma emergente dentro de la cultura de la diversidad, trasladado al ámbito educativo, entra en dificultades, ya que esta es un discurso ideológico, un “(...) slogan de moda de innovación educativa y no como una verdadera transformación en el pensamiento y en la práctica pedagógica que exige otro modo de educación, considerando la diferencia como un valor en el ser humano" (López, 2002, p. 14). Se requiere un cambio paradigmático que logre una relectura de la dinámica cambiante de un nuevo modelo de cultura de escuela que facilite la reconceptualización de la sociedad. Un planteamiento desde la orientación es potenciar la educación inclusiva y la misma inclusividad en todos los procesos educativos (formales o no formales).

Es decir, se necesita una nueva escuela para una nueva civilización, en una sociedad multicontextual y multicultural, donde se fomente el respeto, la tolerancia y la libertad de pensamiento desde los fundamentos de la interculturalidad y las relaciones equitativas. Crear un principio que facilite "(...) construir la cultura de la diversidad frente a la cultura del hándicap (...). La diversidad hace referencia a la identificación de la persona por la persona, por la que cada cual es como es y no como nos gustaría que fuera (identidad). Este reconocimiento es precisamente lo que configura la dignidad humana. (...) La diferencia es la valoración (...) de la diversidad y es precisamente en esta valoración donde hay varias manifestaciones ya sean de rechazo (antipatía, xenofobia, racismo, intolerancia...) (...) como valor" (López, 2002, p. 26).

En una sociedad multicontextual y multicultural, la disciplina de la orientación debe responder no solo a compartir nuevos modelos de sociabilidad, sino, además, aspirar a la "(...) asunción de nuevos estilos cognitivos basados en la relatividad de los puntos de vista y sobre su posible descentramiento. La educación intercultural, la educación para la mundialidad, no se puede prescindir una ética de la responsabilidad individual y colectiva (...). La naturaleza es diversa y no hay cosa más genuina en el ser humano que la diversidad (...)" (López, 2002, p. 27).

Sostenemos que la escuela del nuevo siglo debe estar sustentada en un modelo educativo de equidad, inclusión, justicia social y total respeto e inteorización de la diversidad como forma de vida, es decir, responde a un lugar donde nadie sea excluido y todos y todas tengan un espacio que ocupar, un sistema con vínculos en donde la igualdad de oportunidades y las relaciones entre los sujetos de la educación (padres y madres de familia, docentes, discente, gestores, entre otros) sean armoniosas, proactivas y partícipes de los mismo procesos. En estas relaciones, las diferencias sociales, económicas, de género, de funcionamiento, de salud, etc., no se conviertan en discriminaciones, sino en un principio de igualdad consistente en ofrecer 
las mismas oportunidades educativas a todas las estudiantes y a todos los estudiantes desde los derechos humanos más fundamentales, derechos que deben cumplirse de forma efectiva en todas las personas beneficiarias de la educación.

Como es evidente, un planteamiento más allá del reducido concepto de "tratamiento de la diversidad" nos remite a una alternativa educativa global, a valorar que la diversidad en el aula es lo único humanamente posible, y que la diversidad no hace referencia a que haya "minorías" o estudiantes con necesidades educativas especiales como grupo excluido (hay dos sexos; diferentes intereses; diversas formas de pensar, de actuar; puede haber diferentes culturas; pueden existir estudiantes con historias familiares diversas, con situaciones que pueden suponer un problema en los sistemas educativos. Sin embargo, esto no niega que las diferencias entre los seres humanos y la riqueza de las diferencias es una forma de avanzar, de crecer y enriquecer todo proceso educativo, social, sistema de valores, y desarrollo de destrezas y habilidades; de acuerdo con esto, la heterogeneidad en la diversidad es un elemento enriquecedor en todos los procesos del ser humano.

De ahí que se debe plantear la necesidad de desarrollar el trabajo escolar de tal manera que todos y todas posean las oportunidades de avanzar desde la aceptación de la propia identidad, autopercepción, autoconocimiento y reafirmación de las fortalezas y destrezas personales-sociales que potencian las acciones para la intervención en la equidad de las propias limitaciones; el reconocimiento de la persona en la validación de todas sus expresiones. Por tanto, es un reto del mismo colectivo docente desarrollar líneas de pensamiento a favor de la diversidad como marco de acción, y no acciones que favorezcan implícitamente un sesgo a favor de grupos sociales y culturales en contra de otros en función de valores, evaluación de los aprendizajes, poder, situación económica, etc.

Este reto educativo visualiza la diversidad en los procesos educativos desde el respeto a la individualización del aprendizaje y enseñanza, sin atropellar la integridad de la persona en sus estilos y ritmos de aprendizaje. Es conveniente dejar claro que los aprendizajes más relevantes en el ser humano se producen con los demás y no de forma aislada o puramente individual: "(...) el proceso de saber es un proceso social cuya dimensión individual no puede ser olvidada o siquiera subestimada (...)" Freire, 2009, p.135).

En estos procesos, la individualización es entendida desde la construcción del propio proceso de aprendizaje. Esto, además de imposible en la práctica, constituye una segregación de física y psicológica con el resto del grupo aprendiente, por lo que se considera que individualizar no es la manera más apropiada de trabajar con la diversidad, sino la construcción social del conocimiento desde las riquezas individuales y las relaciones dialógicas en la educación.

A partir de este planteamiento emergente de la diversidad en todos los procesos pedagógicos, y ante un mundo cambiante y complejo, la orientación como disciplina debe crear apertura de diálogo para poder comprender la misma diversidad e integrarla a la vida de cada ser humano. Diversidad no es un anexo a nuestra existencia, sino una forma de vida que se enriquece con las necesidades y potencialidades de cada ser humano, de cada sociedad, 
URL: http://www.una.ac.cr/educare

de cada sistema, etnia, grupo, etc. Es un continuum de la vida misma, un proceso no estático; implica el reconocimiento de las personas como seres humanos, como riqueza humana que ofrece el cambio y la responsabilidad de todos y de todas.

La diversidad ha de reflejarse, sentirse, vivirse no solo en el discurso, sino también en la cotidianidad (como ideológica manifestación cultural), con coherencia entre ambas: discursocotidianidad; y en la práxis y reflexión constante de la labor del profesional en orientación.

\section{Referencias}

Devalle, A. y Vega, V. (1999). La huella de la escuela abierta a la diversidad (Cap. 1). En A. Devalle y V. Vega (Auts.). Una escuela en y para la diversidad. El entramado de la diversidad (pp. 23-59). Buenos Aires, Argentina: Aique.

Essomba, M. À. (2006). Liderar escuelas interculturales e inclusivas. Equipos directivos y profesora ante la diversidad cultura y la inmigración. España: Graó.

Freire, P. (1973). ¿Extensión o comunicación? La concientización en el medio rural. España: Siglo Veintiuno Editores. Freire, P. (2009). Cartas a quien pretende enseñar. Argentina: Siglo XXI.

García, A. y Sáez, C. (1998). Del racismo a la interculturalidad. Competencias de la educación. Madrid: Narcea.

Gimeno, J. (2005). La educación que aún es posible. España: Morata.

López, M. (2002). Ideología, diversidad y cultura: Una nueva escuela para una nueva civilización. En Fundación Claudina Thévenet (Ed.), Equidad y calidad para atender la diversidad (Primer Congreso Internacional de Integración de niños con discapacidad en la escuela común). Argentina: Espacio Editorial.

Cómo citar este artículo, según APA:

Artavia, C. y Fallas, M. A. (2012). Orientación y diversidad: Por una educación valiosa para todos y todas. Revista Electrónica Educare, 16 (Especial), 47-52. Consultado de http://www.revistas.una.ac.cr/index.php/EDUCARE/issue/view/418

Nota: Para citar este artículo en otros sistemas puede consultar el hipervínculo "Como citar el artículo" en la barra derecha de nuestro sitio web: http://www.revistas.una.ac.cr/index.php/ EDUCARE/index 